\title{
Provokativer Humor in der Begleitung eines Ambulanzprojektes Ein lösungsorientiertes Instrument zur schnellen Veränderung
}

Gute Strukturen zu wahren und Weiterentwicklung auszubalancieren ist eine Kernaufgabe von Führung. Schnelle, leicht empfundene Veränderungen scheinen dabei schwer vorstellbar. Oder geht das systematisch?

Einen anderen uneigennützig zu begleiten, sich mehr zuzutrauen, seine Fähigkeiten besser zu nutzen und so seine Bedürfnisse besser zu befriedigen, bedarf einer verantwortungsvollen Auseinandersetzung mit der Person und ihren Motivatoren. Der provokative Humoransatz kann dabei, richtig eingesetzt, ein lösungsorientiertes Instrument zur schnellen Veränderung sein. Vom Anwender fordert dies analytische Kompetenz, emotionale Beobachtungsgabe im Umgang mit Menschen, Vertrauen in die eigene Intuition - und mitunter ungewöhnliche Reaktionen. Das Gegenüber merkt zumeist intuitiv, ob Sie es gut mit ihm meinen und sich in seinen
Blickwinkel einfühlen können. Die Grundvertrauensbasis ist unabdingbare Voraussetzung dafür, im anderen Menschen positive Kräfte herauszufordern, sodass der provokative Humoransatz effektiv wirken kann.

Im Management von Veränderungsprojekten vereinfacht das Beherzigen dieser Spielregeln den Prozess ungemein. Veränderungen bedeuten - im Großen wie im Kleinen - ein Loslassen von gewohnten und daher „sicheren“ Strukturen. Fehlt der Mut hierzu, begrenzen wir uns in unseren Entwicklungsperspektiven ungemein stark, was vielleicht sogar zu Demotivation und einem Verlust der Ar-

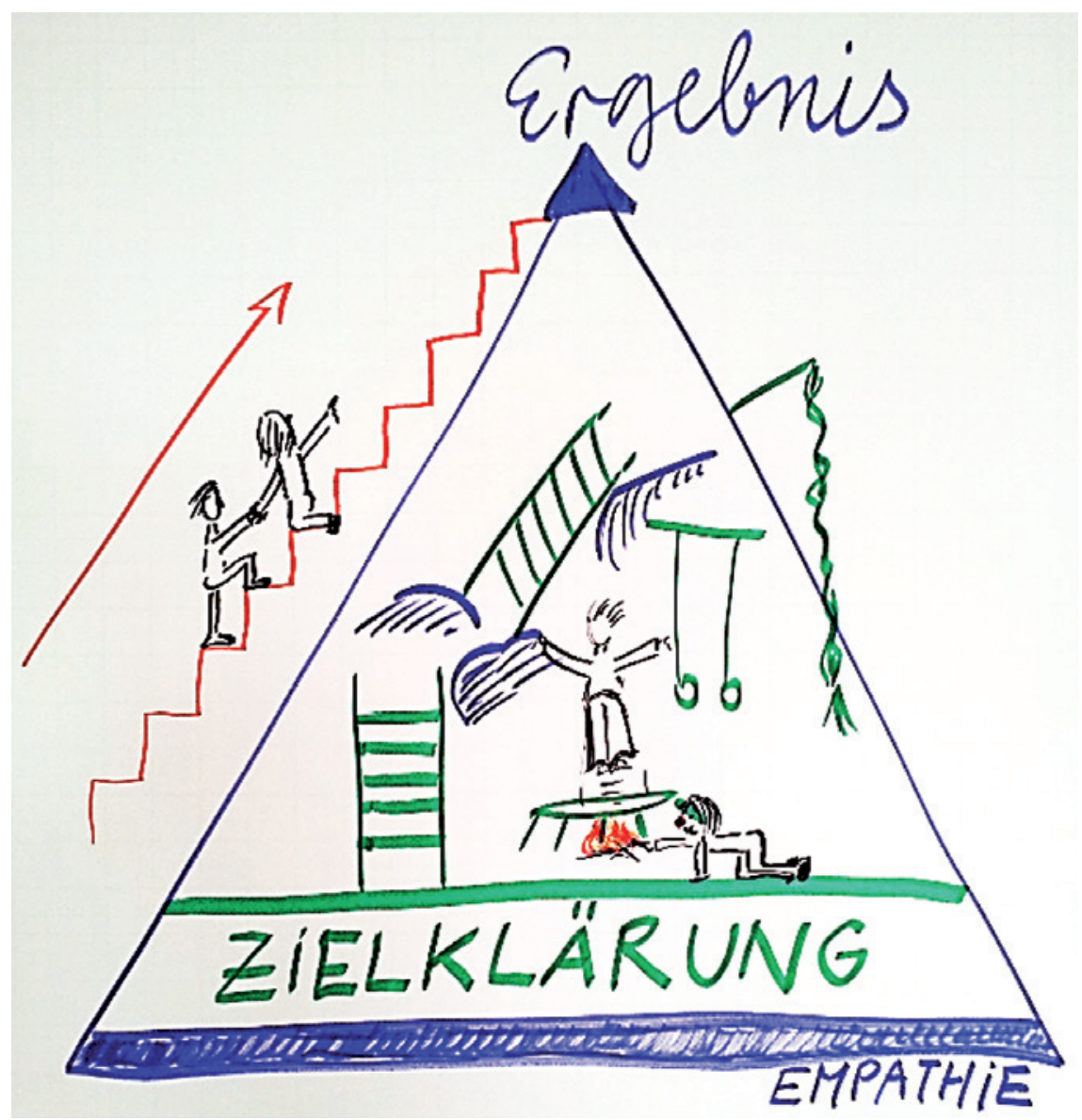

Abb. 1 Der Weg der Veränderungsbegleitung.

beitsfreude führt. Je geringer die Bereitschaft zu Veränderungen und persönlichem Einsatz ist, umso ausgeprägter werden Blockaden, die dazu führen, jede Selbstverantwortung abzugeben. Ist diese Spirale erst einmal in Gang, wird es immer schwieriger, eingefahrene Blickwinkel wieder zu erweitern. Es ist also dringend nötig, der Problemlast eine dosierte Leichtigkeit entgegenzusetzen.

Die Arbeitswelt ist gespickt mit Problemen: Konflikte auf Leitungsebene, Konflikte im Team, Konflikte zwischen Team und Leitung. Diese nicht produktiv aufzulösen lastet dem Unternehmen massiv unnötige Kosten auf. Entsprechend sind professionelle Anstöße von außen hilfreich, die intelligent, belebend, z.T. sogar gepfeffert sind und zu schneller Veränderung motivieren. Im Idealfall kann schon eine einzige pointierte Strategieklausur den Stein ins Rollen bringen. Umfangreichere Begleitungen wirken demgegenüber nachhaltiger, wie etwa nach einer fundierten Auseinandersetzung mit dem Zielbild des Auftraggebers, gemeinsam mit den Nutzern ein Umsetzungskonzept zu konsentieren oder ein Veränderungsprojekt bis zum Abschluss der ersten Umsetzungsphase $\mathrm{zu}$ begleiten. Gemein ist den Ansätzen, dass die vielfach vernetzten Konflikte nicht losgelöst von konkreten Sachthemen und Rollenerwartungen sind und damit eine produktive Arbeitsebene bieten, wie z.B. der im Folgenden dargestellte Erfahrungsbericht.

Projekterfahrung in der Umsetzung eines Veränderungsprojektes Der Projektauftrag betraf die administrative Zusammenlegung mehrerer Teilambulanzen zu einer zentralen Organisation. Die Organisation sollte daraufhin bereits kurzfristig im Baubestand ausgerichtet werden. Die neue Aufbau- und Ablauforganisation soll später mit einer modernen Raum- und Funktionsplanung in einem Neubau verknüpft werden.

Bereits vorher wurden Projektvorhaben gestartet, die nicht zum gewünschten Erfolg führten und insofern verbrannte Erde hinterließen. Es kristallisierte sich als wei- 
tere Erschwernis der Ausgangslage heraus, dass das Projekt alleine durch eine Leitung vor Ort und ein Projektcoaching im Hintergrund, aber auch der direkten Unterstützung durch die oberste Führungsebene bewältigt werden musste.

Ein strukturiertes, phasenweises Projektvorgehen und Rückfallsperren wurden durch ein „agiles“ wöchentliches Projektmanagement sichergestellt. Aufgabe der Projektleitung war es zum einen, vor Ort die Ambulanzmitarbeiter der verschiedenen Fachambulanzen zu einem Team zusammenzuführen, die Neuordnung und Erweiterungen ihrer Aufgabenzuordnungen vorzunehmen und ein systematisches Ausfallmanagement sicherzustellen. Über diesen Hebel sollten Effizienzpotenziale gehoben und damit sowohl die Qualität der Leistungserbringung als auch die wirtschaftliche Tragfähigkeit der Ambulanz verbessert werden. Zum anderen waren die Klinikdirektoren der verschiedenen Fachabteilungen, die zunächst nicht den Nutzen in der Aufgabe ihrer eigenen Teilambulanzen sahen, von der Einrichtung einer zentralen Ambulanzstruktur zu überzeugen.

\section{Der Weg der Veränderung}

Der Durchbruch für die eigentliche Veränderung betrifft eine menschliche Er- fahrung jenseits der bloßen Ratio. Diese betrifft die innersten Bedürfnisse des Menschen bis hin zur Erfahrung, dass alles miteinander verbunden ist. So funktioniert Lernen selten auf dem geraden Weg zum Ziel, sondern in Schleifen (Abb. 1).

Während der Projektlaufzeit wurden Blockaden für die Erreichung des Veränderungsziels auf allen Ebenen in den Projektmanagementtreffen reflektiert und durch die Projektleitung vor Ort unmittelbar und wertschätzend mit den Betroffenen thematisiert. Humor, der an den Emotionen und Bedürfnissen ansetzte, machte es auf angenehm leichte Weise möglich, Menschen in zahlreichen Einzelgesprächen zur Veränderung zu motivieren. Wenn ein kleines Kind nicht einen weiten Weg laufen will, dann werden Eltern erfinderisch und betreten den „Spielegarten“, um ihr Kind Schritt für Schritt auf dem Weg zu motivieren. Wer an dieser Stelle etwa das „Ein Hut, ein Stock, ein Regenschirm“ aus der Trickkiste zieht, motiviert mit sofortiger Belohnung für das Kind. Und im Handumdrehen ist ein gutes Stück des Weges zurückgelegt. Dass diese Schleifen anfangs etwas länger dauern, wird bewusst in Kauf genommen. Die Motivation, die entsteht, überwiegt nachhaltig den kurzfristigen Zeitauf- wand. So etwa soll die Führungskraft jeden Einzelnen aus ihrem Team an die Hand nehmen und auf dem Weg begleiten.

Motivierte Mitarbeiter und Führungskräfte wurden im Projekt aktiv für verschiedene Projektteilziele als Promotoren eingebunden. Das Ambulanzteam hat sich wiederholt durch schwierige Zeiten gekämpft, wobei der Zusammenhalt durch eine lockere und vertrauensvolle Atmosphäre gestärkt werden konnte. Mut für Neues, Verlässlichkeit, Lösungsorientierung, den Dialog auch in Konfliktsituationen zu suchen, haben sich dabei als wichtige Kernkompetenzen des Teams herauskristallisiert.

Um die Mitarbeiter auf ihr neues Aufgabenspektrum vorzubereiten und sie in den Veränderungsprozess einzubinden, wurden als Quick Wins zunächst eine Regelkommunikation im Team eingeführt, regelmäßig stärkenorientierte Mitarbeitergespräche geführt, der effektiv verfügbare Personalstand und eine stärken- und bedarfsorientierte rollierende Diensteinsatzplanung aufgesetzt.

Eine Analyse der Ambulanzabläufe in den Teilambulanzen bezweckte, die vorhandenen Ressourcen zu erkennen und Blockaden gegenüber effektiveren Abläufen aufzudecken. Verschiedene Kennzahlen

Tab. 1 Schnelle Veränderung mit provokativem Humoransatz: Arbeiten auf 3 Ebenen.

\section{Provokation}

\section{Ergebnisprovokation in 3 Stufen}

1 Herausforderung, deren Bewältigung Überwindung bedarf, dem Betroffenen zugetraut wird, ihm neue Grenzerfahrungen verschafft

2 Erwartungsdruck ad absurdum führen

3 Erfolg und Spaß bei Musterdurchbrechung erleben

\section{Schwere Probleme}

1 Lustvolles Erkunden von Stolpersteinen und Schwierigkeiten

2 Direkt, ungeniert und bewertungsfrei darauf eingehen, was der Klient verdeckt

\section{Ergebnis}

Zunehmendes Zutrauen in eigenen Potenziale und in die therapeutische Kompetenz

Veränderung des Blickwinkels, Widerstand gegen eigene Blockaden umleiten

Erreichen einer neuen Erfahrungsebene, Überwinden von Angst und Unsicherheit

Humor

\section{Leicht machen}

Humor und Lachen lösen die Stimmung

Mit humorvoller Distanz kann Widerstand direkt angesprochen, der Finger auf-die-Wunde gelegt werden, Neubewertung wird möglich

Vertrauensbasis

Je mehr Überschuss auf dem Vertrauenskonto, umso mehr kann auf den anderen Ebenen gearbeitet werden. Arbeitsebenen

\section{Vertrauensbildung}

„Rückenstärkung“ erfahren

Haltung und v. a. Ausdrücken von Wohlwollen und Wertschätzung, nonverbale Vermittlung wichtiger als Inhalte, Verbindung etwa über leichte Berührung herstellen

2 Führen über Stolpersteine durch „Pacing and Leading“ (auf die gleiche Ebene begeben, „nichts Besseres sein“, an die Hand nehmen)

3 Die Ressourcen trägt die Organisation in sich, gut zuhören statt langer Analysen: Die persönlichsten Themen sind die universellsten
Kleine, leichte, gemeinsame Schritte Zukunftsbild des Klienten gestalten z.B. Untermauerung durch Zahlen Stärken-/ Ressourcenfokus als Lösungsweg: Selbstbewusstsein stärken 
zur Prozesssteuerung wurden erhoben, so z.B. die Arzt-Patienten-Kontakte und deren Dauer. In einem Organisationshandbuch wurden sukzessive die teils divergierenden Vorstellungen der unterschiedlichen Fachambulanzen bezüglich der Prozessabläufe über gemeinsame Soll-Prozesse vereinheitlicht und verbindlich verabschiedet. Im Handbuch sind zahlreiche Maßnahmen zur Verbesserung der Qualität und Effektivität hinterlegt, wie z.B. die Entrümpelung der Papieraktenpflege, Weiterentwicklung der Service- und Rollenorientierung im direkten Umgang mit Patienten und deren Interessensgruppen, zugewandte Bedienung telefonischer Anfragen im Sinne eines Callcentergedankens, Optimierung der laufenden Leistungsabrechnung und des Rechnungsabschlusses, etc. Entsprechend der funktionalen Aufgabenorganisation wurden die Räume des Ambulanzteams mit einem neuen Raumnutzungskonzept hinterlegt. Zusätzlich wurden Räumlichkeiten für eine zentrale Aufnahme für elektive Patienten geschaffen. Ziel ist es, die Wartezeiten in der Aufnahme zu minimieren. Der Prozess wird über eine Aufrufanlage, einen elektronischen Ambulanzkalender und die gezielte Koordination mit den zentralen Auskunftsstellen, insbesondere der Pforte, unterstützt.

Diese Sachthemen lassen sich - auch in dem hier beschriebenen Umfang - schnell bearbeiten, wenn die Menschen sich einlassen und mit auf die Reise gehen. Dort wo Widerstände aufkeimen, gilt es dann, sich auf das Bild des Betroffenen ganz einzulassen, es zu erfassen, Perspektiven zu ändern, schädliche Glaubenssätze aufzudecken. All dies kann schmerzhaft sein und braucht dementsprechend Zeit zum „Schleifendrehen“. Eine unbedingte Haltung der Empathie ist unabdingbar, eine „zu weiche“ Kommunikation, reines GutZureden ist jedoch nicht ausreichend. Ziel ist es, gemeinsam auf den Weg zu gehen statt eine Haltung des ,jetzt macht ihr mal!“ an den Tag zu legen. Der Weg ist die Aufgabe an sich: es gilt den Fokus zu verlagern, was hat der andere davon und mit positiver Überraschung den Spaß und den Mut zur Veränderung hervorzulocken. Liebevolle Provokation kann den letzten Anstoß geben - sofern sichergestellt ist, dass dies beim anderen nicht als Zeichen der Respektlosigkeit aufgefasst wird. Ist der Wunsch beim Nutzer selbst geweckt, das Veränderungsziel zu errei- chen, dann ist der Rest schnell gemacht: Muster erkennen, durchbrechen, verrückte Impulse einstreuen, neue Routinen eintrainieren.

\section{Unmittelbare Projektergebnisse}

Zentrale Erfolge der Projektarbeit umfassen die Integration aller beteiligten Klinikdirektoren in die Projektorganisation und das Herausbilden eines gemeinsamen Qualitätsgedankens. Die neue zentrale Ambulanz verfügt nach einer Veränderung der Aufbauorganisation über eine wirksame Führung, die sowohl eine Einbindung der Klinikdirektoren als auch die Führung des Ambulanzteams unter einer zentralen Leitung ermöglicht. Dies bildete die Grundlage dafür, weitere Schritte hin zu zentralisierten Strukturen sowie zu einem übergreifenden Termin- und Ressourcenmanagement zu gehen.

Die Behandlungszahlen sind in einigen Teilambulanzen konstant bzw. in anderen Teilambulanzen gegenüber dem Vorjahr gestiegen und werden mit insgesamt weniger Mitarbeitern im Funktionsbereich administrativ bewältigt. Die Abrechnungsprozesse funktionieren zeitnaher und weniger problembehaftet als in der Vergangenheit. Die Mitarbeiter des Funktionsbereichs werden zudem kontinuierlich und einheitlich geschult in Bezug auf Service- und Dienstleistungsorientierung. So konnten bereits in der ersten Phase der Umstrukturierung sowohl die Servicequalität als auch die Deckungsbeiträge der Ambulanz gesteigert werden.

Nach 9 Monaten Projektbegleitung haben sich die Erfolge weiter stabilisiert. Durch räumlich-strukturelle, aufbau- und ablauforganisatorische Veränderungen des administrativen Ambulanzbereichs ist eine Rückfallsperre in frühere Verhältnisse garantiert. Das Projekt wurde abgeschlossen und in den Regelbetrieb übertragen. Bemerkenswert ist, mit welcher Leichtigkeit und Geschwindigkeit es im Projekt gelungen ist, zentrale Ambulanzprozesse einzuführen, umzusetzen und zu stabilisieren - und dabei ein hohes Committment und eine hohe Verbindlichkeit der ärztlichen Leistungsträger zu erreichen. Die Wirkung des beherzten Einsatzes des provokativen Humors auf Augenhöhe in Einzelgesprächen und in den monatlichen Treffen des Lenkungsausschusses kann nur mehrfach unterstrichen werden. Hier kommt der Projektleiterin die klare Rollendefinition zugute: Sie hat die Entscheidungen des Len- kungsausschusses umzusetzen, aber keine Eigeninteressen daran. Dadurch, dass sie Entscheidungsvorlagen und persönliche ressourcenorientierte Lösungsvorschläge vorträgt, ohne an diese inhaltlich und emotional gebunden zu sein, steht ihr das volle Repertoire des provokativen Humoransatzes zur Verfügung. Und der verfehlt seine Wirkung in der Projektleitung ebenso wenig wie in der Therapie.

\section{Literatur}

1 Eberts E, Ruhl S. Provokativer Humoransatz - Menschen in Organisationen für Veränderung motivieren, In: Die besten Ideen für mehr Humor: Erfolgreiche Speaker verraten ihre besten Konzepte und geben Impulse für die Praxis. Sabine Asgodom (Hrsg.), Offenbach: Gabal-Verlag 2013: 80-90

2 Farrelly F, Brandsma JM. Provocative Therapy. Cupertino: Meta 1974

3 Höfner E. Das wäre doch gelacht! Humor und Provokation in der Therapie. Reinbek: Rowohlt. 1995

4 Höfner E. Glauben Sie ja nicht, wer Sie sind! Grundlagen und Fallbeispiele des Provokativen Stils. Heidelberg: Carl-Auer 2011

5 Keßler A. Im Elfenbeinturm. Oder: Folge ich meinen eigenen Ratschlägen? In: Kernberg OF, Dulz B, Eckert J (Hrsg.). Wir: Psychotherapeuten über sich und ihren „unmöglichen" Beruf. Stuttgart: Schattauer Verlag 2005: 515-521

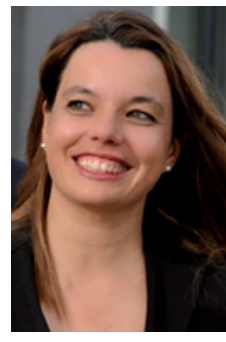

Korrespondenz Dr. rer. pol. Elke I. Eberts Ruhl Consulting AG Harrlachweg 1 68163 Mannheim Tel: 0621/32886490 E-Mail: info@ruhl-consulting.de www.ruhl-consulting.de

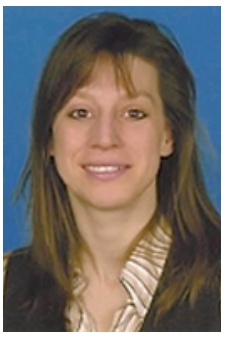

Dr. med. Astrid S. Keßler Psychotherapeutische Privatpraxis Think-Praxis U 6, 12 68161 Mannheim E-Mail: kessler@thinkpraxis.de www.think-praxis.de 